\title{
Synthesis and $\sigma_{1}$ Receptor Binding of Halogenated N,N'- Diphenethylethylenediamines
}

\author{
Jonathan M. Fitzsimmons ${ }^{1,5}$, John R. Lever ${ }^{2,3,5}$ and Susan Z. Lever ${ }^{1,4 *}$ \\ ${ }^{1}$ Departments of Chemistry, University of Missouri - Columbia, Columbia, MO, USA \\ ${ }^{2}$ Departments of Radiology, University of Missouri - Columbia, Columbia, MO, USA \\ ${ }^{3}$ Departments of Medical Pharmacology and Physiology, University of Missouri - Columbia, Columbia, MO, USA \\ ${ }^{4}$ University of Missouri Research Reactor Center, University of Missouri - Columbia, Columbia, MO, USA \\ ${ }^{5}$ Research Service, Harry S. Truman Memorial Veterans' Hospital, Columbia, MO, USA
}

\begin{abstract}
Eight halogenated $N, N^{\prime}$-diphenethylethylenediamines were synthesized, characterized and evaluated for $\sigma_{1}$ receptor binding affinity in vitro. Measurements of lipophilicity also were obtained. The substitution pattern on one of the aromatic rings remained constant as 3,4-dichloro, while the substituents on the other aromatic ring were varied to include fluorine, bromine or iodine in either the 2-, 3- or 4- positions. Two main structure activity relationships were observed. First, halogen substitution on the 3- or 4-positions of the aromatic ring conferred higher binding affinities ( $K_{\mathrm{i}}$ values $\left.6.35-15.82 \mathrm{nM}\right)$ than the corresponding substitutions at the 2-position ( $K_{\mathrm{i}}$ values $\left.12.08-43.15 \mathrm{nM}\right)$. Second, derivatives containing either a bromo or fluoro substituent at a given position showed higher $\sigma_{1}$ receptor binding affinities than derivatives with a corresponding iodo substituent. The data indicate that $\sigma_{1}$ receptor affinity for this structural series is sensitive to steric bulk at the 2-position. $\log k^{\prime}$ measurements for the halogenated $N, N^{\prime}-$ diphenethylethylenediamines were determined by high performance liquid chromatography, and varied from 2.54 - 3.71. In particular, the 3-fluoro analog exhibited a $\log k^{\prime}=2.54$ accompanied by a $\sigma_{1}$ receptor $K_{i}=7.8 \mathrm{nM}$. These novel $\mathrm{N}, \mathrm{N}^{\prime}$-diphenethylethylenediamines warrant further investigation in behavioral assays, and radiolabeled versions may prove suitable for in vivo studies of $\sigma_{1}$ receptors.
\end{abstract}

Keywords: Sigma receptor; Binding studies; Structure activity relationships; lipophilicity

\begin{abstract}
Abbreviations: SAR: Structure-activity relationship; SPECT: Single photon emission computed tomography; PET: Positron emission tomography; HPLC-MS: High performance liquid chromatographymass spectrometry; ESI-MS: Electrospray ionization mass spectrometry; TFA: Trifluoroacetic acid; DCC: N, $\mathrm{N}^{\prime}$-dicyclohexylcarbodiimide; DCU: N,N'-dicyclohexylurea; THF: Tetrahydrofuran; NHP: N-hydroxyphthalimide; MOPS: 3-(N-morpholino)propanesulfonic acid; Tris-HCl: Tris(hydroxymethyl)aminomethane hydrochloride; BSA: Bovine serum albumin; SEM: Standard error of the mean; BD1047: N-[2-(3,4-dichlorophenyl)ethyl]-N-methyl-2(dimethylamino)ethylamine; BD1063: 1-[2-(3,4-dichlorophenyl) ethyl]-4-methylpiperazine; $\quad$ YZ069: N-phenylpropyl-N' ${ }^{\prime}-(3,4-$
\end{abstract} dichlorophenethyl)piperazine.

\section{Introduction}

Sigma $(\sigma)$ receptors can be classified into two distinct subtypes, $\sigma_{1}$ and $\sigma_{2}$, based upon their relative protein sizes, tissue and cellular distributions, and pharmacological / biochemical profiles [1-5]. There is considerable current interest in $\sigma_{1}$ receptors as therapeutic targets for multiple central nervous system disorders, including schizophrenia, depression, anxiety, Alzheimer's disease and stroke [5-9]. Further, there is a growing body of evidence that $\sigma_{1}$ receptor ligands, particularly selective antagonists, reduce the reinforcing effects of alcohol [10] and attenuate the behavioral effects of psychostimulant drugs of abuse $[1,5,11-15]$. The $\sigma_{2}$ receptors also may play a modest role in mitigating the actions of abused drugs $[1,15]$, but truly selective $\sigma_{2}$ receptor ligands are just now being identified that might allow definitive discrimination of individual $\sigma$ receptor subtype contributions [15-17].

Over the years, a number of $N, N^{\prime}$-disubstituted ethylenediamines and piperazines have been investigated as $\sigma_{1}$ receptor ligands $[1,15,18]$. Such compounds typically exhibit $K_{\mathrm{i}}$ values of 1 - $10 \mathrm{nM}$ for $\sigma_{1}$ receptors, accompanied by 2 - to 50 -fold selectivities against $\sigma_{2}$ sites. Prototypical ligands include BD1047 and BD1063 (Figure 1) that mitigate cocaineinduced lethality, locomotor activity and conditioned place preference in mice [1]. A series of $N$-benzyl- $N^{\prime}$-benzylpiperazines (Figure 1,1) showed higher affinities for $\sigma_{1}$ receptors, $K_{\mathrm{i}}$ values of $0.39-7.6 \mathrm{nM}$, accompanied by greater selectivities, 13 - to 340 -fold selectivity against $\sigma_{2}$ sites [19]. This active series potently attenuates cocaine-induced convulsions in mice, except for the 3,4-dichloro derivative that unexpectedly behaves as an agonist.

Structural modifications of the ethylenediamine and piperazine scaffolds generally are well tolerated. For instance, $N$-phenylpropyl$N^{\prime}$-phenethylpiperazines, such as YZ069 (Figure 1,2), display $\sigma_{1}$ receptor $K_{\mathrm{i}}$ values of $0.7-3.9 \mathrm{nM}, 2$ - to 22 -fold selectivities against $\sigma_{2}$ sites, and protect mice against cocaine-induced convulsions [20]. Recently, a series of ten $N$-(3-phenylpropyl)- $N$-benzylpiperazines (Figure 1,3) showed $\sigma_{1}$ receptor $K_{\mathrm{i}}$ values of $0.37-2.8 \mathrm{nM}$, with 1.4 - to 52 -fold selectivities against $\sigma_{2}$ sites [21]. Robust quantitative SAR were established for their $\sigma_{1}$ and $\sigma_{2}$ receptor binding, and these compounds profiled as probable $\sigma_{1}$ receptor antagonists based upon an in vitro test using phenytoin as an allosteric modulator of competition against $\left[{ }^{3} \mathrm{H}\right]-(+)$-pentazocine.

In order to gain further insight into $\sigma$ receptor SAR, and to expand the armamentarium of ligands available for biological testing, we have synthesized and characterized a series of eight halogenated $N, N^{\prime}$ diphenethylethylenediamines (Figure 1,4-11), determined $\sigma_{1}$ receptor

*Corresponding author: Susan Z Lever, PhD, Room 125 Chemistry Building University of Missouri - Columbia, 601 South College Avenue, Columbia, MO 65211 USA, Tel: (573) 882-8395; Fax: (573) 882-2754; E-mail: levers@missouri. edu

Received September 22, 2011; Accepted November 08, 2011; Published December 25, 2011

Citation: Fitzsimmons JM, Lever JR, Lever SZ (2011) Synthesis and $\sigma_{1}$ Receptor Binding of Halogenated N,N'-Diphenethylethylenediamines. Medchem 1:102 doi:10.4172/2161-0444.1000102

Copyright: (c) 2011 Fitzsimmons JM, et al. This is an open-access article distributed under the terms of the Creative Commons Attribution License, which permits unrestricted use, distribution, and reproduction in any medium, provided the original author and source are credited. 
<smiles>CN(C)CCN(C)CCc1ccc(Cl)c(Cl)c1</smiles>

BD1047<smiles>[R]1cccc(CN2CCN(Cc3ccccc3)CC2)c1</smiles>

1: $N$-benzyl- $N$ '-benzylpiperazines: $\mathrm{R}=\mathrm{H}$, 3-OMe, 4-OMe, 2-Cl, 3-Cl, 4-Cl, 2,4-di-Cl, 3,4-di-Cl<smiles>c1ccc(CCCN2CCN(CCc3ccccc3)CC2)cc1</smiles>

2: $N$-phenylpropyl- $N$ '-phenethylpiperazines; YZ069, R = 3,4-dichloro<smiles>c1ccc(CCCN2CCN(Cc3ccpcc3)CC2)cc1</smiles>

3: $N$-(3-phenylpropyl)- $N$ '-benzylpiperazines; $\mathrm{R}=2-\mathrm{Br}, 2-\mathrm{NO}_{2}, 3-\mathrm{I}, 3-\mathrm{F}, 3-\mathrm{OCH}_{3}, 3-\mathrm{NO}_{2}$, $4-\mathrm{OCH}_{3}, 4-\mathrm{NO}_{2}, 4-\mathrm{CH}_{3}$<smiles>Clc1ccc(CCNCCNCc2ccccc2)cc1</smiles>
$4, R=2-F$
$7, \mathrm{R}=2-\mathrm{Br}$
$5, R=3-F$
8, $R=3-B r$
9, $R=4-B r$
$10, R=2-1$
$6, R=4-F$
$11, R=4-1$

Figure 1: Structures of representative $\sigma_{1}$ receptor ligands, and the novel series of halogenated $N, N^{\prime}$-diphenethylethylenediamines 4 - 11.

binding parameters in vitro, and measured lipophilicity by HPLC (log $\left.k_{\mathrm{w}}^{\prime}\right)$ and computational $(\mathrm{C} \log \mathrm{P})$ methods. One of the aromatic rings was held constant with a 3,4-dichloro pattern, while the other ring was varied to include fluoro, bromo and iodo substituents at the 2-, 3- or 4- positions. We focused on halogen substitution with a longerterm view toward radiolabeling with fluorine-18 for PET imaging, or radiolabeling with iodine-123 for SPECT imaging [22,23].

\section{Materials and Methods}

\section{General information}

Chemical reagents and HPLC solvents were the best grade available from Aldrich Chemical Co. (Milwaukee, WI), and were used as received unless further noted. Reaction solvents $\left(\mathrm{CH}_{2} \mathrm{Cl}_{2}, \mathrm{CH}_{3} \mathrm{CN}\right.$, THF and benzene) were dried, and freshly distilled under nitrogen before use. Ethylenediamine was distilled from freshly activated, $5 \AA$ molecular sieves, and the heart cut was then distilled from sodium metal (bp 117.0$\left.117.5^{\circ} \mathrm{C}\right) .{ }^{1} \mathrm{H}$ and ${ }^{13} \mathrm{C}$ NMR were performed using ARX-250, DRX-300 or DRX-500 MHz spectrometers (Bruker BioSpin Corp., Westmont, IL). Chemical shifts are reported in ppm $(\delta)$ relative to internal $\mathrm{Me}_{4} \mathrm{Si}$ in $\mathrm{CDCl}_{3}$ unless otherwise stated. Elemental analyses were determined by Atlantic Microlab, Inc. (Norcross, GA). The C, H, N analyses were performed by combustion using automated analyzers, and the accuracy and precision are $\pm 0.3 \%$. ESI-MS analyses were performed on a Finnigan TSQ7000 mass spectrometer (Thermo Finnigan, San Jose, CA). The HPLC-MS analyses utilized a Waters (Milford, MA) C18 Nova Pak column $(3.9 \times 300 \mathrm{~mm})$ with a solvent system comprised of an aqueous phase including $0.1 \%$ TFA and an organic phase including $0.1 \%$ TFA in acetonitrile with the following gradient program: Time $(\mathrm{t})=0 \min 5 \% \mathrm{~B}, \mathrm{t}=2 \min 40 \% \mathrm{~B}, \mathrm{t}=7 \min 40 \% \mathrm{~B}, \mathrm{t}=37 \min 80 \%$ $\left.\mathrm{B}, \mathrm{t}=39 \min 95 \% \mathrm{~B}, \mathrm{t}=45 \min 95 \% \mathrm{~B} .{ }^{3} \mathrm{H}\right]-(+)$-Pentazocine $(36 \mathrm{Ci}$ / mmol) was purchased from Perkin Elmer Life Sciences (Waltham, MA), and fresh-frozen English Hartley guinea pig brains were obtained from Rockland Immunochemicals, Inc. (Gilbertsville, PA). A Brandel R48 manifold (Brandel Instruments, Gaithersburg, MD) was used for receptor binding filtrations. Radioactivity was measured using a Wallac 1409 (Turku, Finland) liquid scintillation counter and OptiPhase HiSafe 2 cocktail (Perkin Elmer) at a tritium efficiency of $45 \%$.

\section{Chemistry}

N-(2-aminoethyl)-2-(3,4-dichlorophenyl)-acetamide (12): 3,4-Dichlorophenylacetic acid $(5.36 \mathrm{~g}, 0.0261 \mathrm{~mol})$ and $\mathrm{N}$-hydroxyphthalimide $(4.36 \mathrm{~g}, 0.0267 \mathrm{~mol})$ were added to a flask containing $\mathrm{CH}_{2} \mathrm{Cl}_{2}(50 \mathrm{~mL})$, treated with a solution of DCC $(7.78$ g, $0.0377 \mathrm{~mol})$ in $\mathrm{CH}_{2} \mathrm{Cl}_{2}(50 \mathrm{~mL})$, and stirred for $30 \mathrm{~min}$. DCU was removed by filtration, and the filtrate added dropwise to neat ethylenediamine $(13.5 \mathrm{~g}, 0.224 \mathrm{~mol})$ over $30 \mathrm{~min}$. The solution was stirred overnight, filtered and treated with $10 \%$ citric acid $(75 \mathrm{~mL})$. The $\mathrm{pH}$ was adjusted to 3 by drop-wise addition of concentrated $\mathrm{HCl}$. The aqueous layer was separated, the organic layer was extracted with water, and the aqueous layers were combined. The aqueous solution was brought to $\mathrm{pH} 12$ with concentrated $\mathrm{NH}_{4} \mathrm{OH}$, and extracted with $\mathrm{CHCl}_{3}$. The extracts were pooled, dried with anhydrous $\mathrm{Na}_{2} \mathrm{SO}_{4}$, filtered and evaporated under reduced pressure. The yellow oil was dissolved in absolute ethanol $(10 \mathrm{~mL})$, and treated with $49 \% \mathrm{HBr}(2 \mathrm{~mL})$ to give 12 as a white salt $(0.34 \mathrm{~g}, 52 \%) .{ }^{1} \mathrm{H}$ NMR $\left(300 \mathrm{MHz}, \mathrm{D}_{2} \mathrm{O}\right): \delta 3.09(\mathrm{t}$, $\left.2 \mathrm{H}, \mathrm{CH}_{2} \mathrm{NH}_{2}\right) ; 3.45$ (t, $\left.2 \mathrm{H},(\mathrm{C}=\mathrm{O}) \mathrm{NHCH}_{2}\right) ; 3.58$ (s, $\left.2 \mathrm{H}, \mathrm{Ar} \mathrm{CH}_{2}\right) ; 7.1$ (d, $1 \mathrm{H}, \operatorname{ArH}) ; 7.461$ (t, 2H, ArH). ${ }^{13} \mathrm{C}$ NMR $\left(75 \mathrm{MHz}, \mathrm{D}_{2} \mathrm{O}^{\mathrm{i}}\right): \delta 36.89$, $38.98,40.96,129.04,130.43,130.46,130.98,131.61,134.86,174.4$. MSESI direct infusion: Theory $\left(\mathrm{M}^{+} \mathrm{m} / \mathrm{z}, \%\right): 246.8,100 ; 248.7,69$. Found $\left(\mathrm{M}^{+} \mathrm{m} / \mathrm{z}, \%\right): 246.0,100 ; 248.0,64$.

General method for synthesis of amides 13-20: The hydrobromide salt of 12 was dissolved in water, treated with $\mathrm{NH}_{4} \mathrm{OH}$, and the free amine extracted with $\mathrm{CH}_{2} \mathrm{Cl}_{2}(3 \times 100 \mathrm{~mL})$. The organic extracts were dried over anhydrous $\mathrm{Na}_{2} \mathrm{SO}_{4}$, filtered and volatiles removed under reduced pressure. The residual oil was dissolved in THF $(50 \mathrm{~mL})$ and 1 equivalent of $\mathrm{Et}_{3} \mathrm{~N}$ was added. Then 1.5 to 2 equivalents of the appropriate acid chloride were prepared by refluxing the halogenated phenylacetic acid with thionyl chloride (2.5 - 10 volume equivalents per gram acid). THF $(2 \mathrm{~mL})$ was added to the acid chloride solution, and volatiles were evaporated under reduced pressure. The residual oil was dissolved in THF, and added drop-wise to the amine solution. The reaction was stirred overnight, and then slowly added to $2 \mathrm{M}$ $\mathrm{HCl}(10 \mathrm{~mL})$. The mixture was concentrated under reduced pressure to approximately $10 \mathrm{~mL}$, and extracted with $\mathrm{CHCl}_{3}(3 \times 20 \mathrm{~mL})$. The organic fractions were pooled, and evaporated under reduced pressure. The remaining oil was dissolved in absolute ethanol, and added dropwise to water to yield a white precipitate that was isolated by filtration, dried and characterized.

$\mathrm{N}$-\{2-[2-(3-Fluorophenyl)-acetylamino]-ethyl $\}-2-(3,4-$ dichlorophenyl)-acetamide (14): The acid chloride generated from 3-fluorophenylacetic acid (0.56 g, 0.0036 moles) was added to a 
solution of 12 ( $0.61 \mathrm{~g}, 0.0019$ moles) and $\mathrm{Et}_{3} \mathrm{~N}$ (0.54 mL, 0.0039 moles) to generate the diamide $(0.34 \mathrm{~g}, 47 \%) .{ }^{1} \mathrm{H}$ NMR $\left(300 \mathrm{MHz}, \mathrm{D}_{6}\right.$-DMSO): $\delta 3.10\left(\mathrm{~s}, 4 \mathrm{H},(\mathrm{C}=\mathrm{O}) \mathrm{NHCH}_{2}\right) ; 3.41\left(\mathrm{~s}, 4 \mathrm{H}, \mathrm{Ar} \mathrm{CH}_{2}\right) ; 7.05$ (d, 2H, $\left.\mathrm{ArH}\right)$; 7.22 (d, 1H, ArH); 7.31 (q, 1H, ArH); 7.51 (d, 1H, ArH); 7.55 (s, 1H, $\mathrm{ArH}) ; 8.10$ (br s, $\left.2 \mathrm{H}, \mathrm{NH}_{2}\right) .{ }^{13} \mathrm{C}$ NMR $\left(75 \mathrm{MHz} \mathrm{D}_{6}\right.$-DMSO): $\delta 40.98$, $41.84,112.97,113.25,115.62,115.90,125.15,129.03,129.52,129.90$, 130.01, 130.22, 130.59, 131.09, 137.39, 139.07, 169.42, 169.72. MS-ESI: Theory $(\mathrm{M}+\mathrm{H} \mathrm{m} / \mathrm{z}, \%): 383.07,100 ; 385.1,64$. Found $(\mathrm{M}+\mathrm{H} \mathrm{m} / \mathrm{z}, \%)$ : $383.09,100 ; 385.0,60$.

$\mathrm{N}$ - $\{2$ - [2-(4-Fluorophenyl)-acetylamino]-ethyl $\}-2-(3,4-$ dichlorophenyl)-acetamide (15): The acid chloride generated from 4-fluorophenylacetic (1.01 g, 0.0065 moles) acid was added to a solution of 12 ( $0.64 \mathrm{~g}, 0.0019$ moles $)$ and $\mathrm{Et}_{3} \mathrm{~N}(0.87 \mathrm{~mL}, 0.0062$ moles $)$ to generate the diamide $(0.69 \mathrm{~g}, 93 \%) .{ }^{1} \mathrm{H}$ NMR $\left(300 \mathrm{MHz}, \mathrm{D}_{6}-\mathrm{DMSO}\right)$ : $\delta 2.18\left(\mathrm{~m}, 4 \mathrm{H},(\mathrm{C}=\mathrm{O}) \mathrm{NHCH}_{2}\right) ; 3.38\left(\mathrm{~d}, 4 \mathrm{H}, \mathrm{Ar} \mathrm{CH}_{2}\right) ; 7.09$ (t, 2H, $\left.\mathrm{ArH}\right)$; 7.23 (m, 3H, ArH); 7.52 (t, 2H, ArH); 8.07 (br d, 2H, NH). ${ }^{13} \mathrm{C}$ NMR $(75$ $\mathrm{MHz}, \mathrm{D}_{6}$-DMSO): $\delta 38.72,38.84,41.37,41.70,115.07,115.35,129.42$, 129.91, 130.61, 130.97, 131.12, 131.22, 131.48, 132.80, 169.80, 170.54 . HPLC-MS, retention time $=21.7 \mathrm{~min}$. Theory $(\mathrm{M}+\mathrm{H} \mathrm{m} / \mathrm{z}, \%): 383.0$, $100 ; 384.0,19 ; 385.0,64$. Found $(\mathrm{M}+\mathrm{H} \mathrm{m} / \mathrm{z}, \%): 383.0,100 ; 384.0,13$; $385.0,69$.

$\mathrm{N}$ - $\{2$ - [2-(2-Bromophenyl)-acetylamino]-ethyl $\}-2-(3,4-$ dichlorophenyl)-acetamide (16): The acid chloride generated from 2-bromophenylacetic acid ( $1.57 \mathrm{~g}, 0.0073$ moles) was added to a solution of $\mathbf{1 2}$ ( $1.5 \mathrm{~g}, 0.0046$ moles) and $\mathrm{Et}_{3} \mathrm{~N}$ (1.03 mL, 0.0074 moles) to generate the diamide (1.48 g, $72 \%)$. ${ }^{1} \mathrm{H} \mathrm{NMR}\left(300 \mathrm{MHz}, \mathrm{CDCl}_{3}\right): \delta$ 3.5-3.6 (s and $\left.\mathrm{m}, 8 \mathrm{H}, \mathrm{CH}_{2}(\mathrm{C}=\mathrm{O}) \mathrm{NHCH}_{2}\right) ; 6.5$ (br s, $1 \mathrm{H}, \mathrm{NH}$ ); 7.1-7.2 (d, $1 \mathrm{H} \mathrm{NH}) ; 7.3-7.4$ (m, 1H, ArH); 7.37-7.40 (m, 3H, ArH); 7.5-7.6 (dd, 1H, ArH); 7.69-7.73 (m, 1H, ArH); 7.95-7.99 (m, 1H, ArH). ${ }^{13} \mathrm{C}$ NMR $\left(125 \mathrm{MHz}, \mathrm{CDCl}_{3}\right): \delta 37.29,39.78,42.52,43.93,49.77,124.98,127.60$, $128.15,128.76,128.89,129.25,130.66,131.26,131.86,132.83,133.20$, $134.40,170.48,171.08$. HPLC-MS, retention time $=8.5 \mathrm{~min}$. Theory $(\mathrm{M}+\mathrm{H} \mathrm{m} / \mathrm{z}, \%): 443.9,100 ; 441.9,62 ; 445.9,45$. Found $(\mathrm{M}+\mathrm{H} \mathrm{m} / \mathrm{z}, \%)$ : $443.0,100 ; 441.0,65 ; 445.1,43$.

$\mathrm{N}$-\{2-[2-(3-Bromophenyl)-acetylamino]-ethyl $\}-2-(3,4-$ dichlorophenyl)-acetamide (17): The acid chloride generated from 3-bromophenylacetic acid ( $1.3 \mathrm{~g}, 0.0062$ moles) was added to a solution of 12 (1.6 g, 0.0048 moles) and $\mathrm{Et}_{3} \mathrm{~N}(0.86 \mathrm{~mL}, 0.0062$ moles) to generate the diamide (0.98 g, 46\%). ${ }^{1} \mathrm{H}$ NMR $\left(300 \mathrm{MHz} \mathrm{CDCl}_{3}\right): \delta 3.35(\mathrm{t}, 4 \mathrm{H}$, $\left.(\mathrm{C}=\mathrm{O}) \mathrm{NHCH}_{2}\right) ; 3.46$ (d, 4H, $\left.\mathrm{Ar} \mathrm{CH}_{2}\right) ; 6.10$ (br s, $\left.1 \mathrm{H}, \mathrm{NH}\right) ; 6.26$ (br s, $1 \mathrm{H} \mathrm{NH}) ; 7.07$ (dd, 1H, ArH); 7.17 (d, 1H, ArH); 7.22 (d, 1H, ArH); 7.37 (d, $1 \mathrm{H}, \mathrm{ArH}) ; 7.40-7.46$ (m, 3H, ArH) ${ }^{13} \mathrm{C}$ NMR $\left(75 \mathrm{MHz}, \mathrm{CDCl}_{3}\right)$ $\delta 40.07,40.50,42.46,43.06,122.90,128.0,128.7,130.5,130.6,130.7$, $131.2,132.3,134.7,136.7,170.9,171.63$. MS-ESI: Theory $(\mathrm{M}+\mathrm{H} \mathrm{m} / \mathrm{z}$, $\%): 444.9,100 ; 442.9,62$. Found $(\mathrm{M}+\mathrm{H} \mathrm{m} / \mathrm{z}, \%): 444.7,81 ; 442.7,43$. Theory $\left(\mathrm{M}+\mathrm{NH}_{4} \mathrm{~m} / \mathrm{z}, \%\right): 462.0,100 ; 464.0,44$. Found (m/z, \%): 462.9, $100 ; 464.9,74$.

$\mathrm{N}-\{2-[2-(4-\mathrm{Bromophenyl})$-acetylamino]-ethyl $\}-2-(3,4-$ dichlorophenyl)-acetamide (18): The acid chloride generated from 4-bromophenylacetic acid (2.23 g, 0.0104 moles) was added to a solution of 12 (2.9 g, 0.0088 moles) and $\mathrm{Et}_{3} \mathrm{~N}$ (1.59 mL, 0.0114 moles) to generate the diamide $(2.5 \mathrm{~g}, 65 \%) .{ }^{1} \mathrm{H}$ NMR $\left(300 \mathrm{MHz}, \mathrm{CDCl}_{3}\right): \delta 3.3$ (s, $\left.4 \mathrm{H},(\mathrm{C}=\mathrm{O}) \mathrm{NHCH}_{2}\right) ; 3.4$ (d, $\left.4 \mathrm{H}, \mathrm{Ar} \mathrm{CH}_{2}\right) ; 5.9$ (br s, $\left.1 \mathrm{H}, \mathrm{NH}\right) ; 6.2(\mathrm{br}$ s, $1 \mathrm{H}, \mathrm{NH}) ; 7.0(\mathrm{~m}, 3 \mathrm{H}, \operatorname{ArH}) ; 7.36(\mathrm{~d}, 1 \mathrm{H}, \operatorname{ArH}) ; 7.41(\mathrm{~d}, 1 \mathrm{H}, \mathrm{ArH})$; 7.48 (d, 2H, ArH). ${ }^{13} \mathrm{C}$ NMR $\left(75 \mathrm{MHz}, \mathrm{CDCl}_{3}\right): \delta 39.88,40.01,40.51$, $40.63,42.48,42.52,42.90,42.94,121.52,128.75,130.75,131.06,131.22$, $131.57,132.10,132.81,133.48,134.80,170.78,171.77$. MS-ESI(+): Theory $(\mathrm{M}+\mathrm{Na} \mathrm{m} / \mathrm{z}, \%): 466.9,100 ; 464.9,62 ; 468.9,45$. Found $(\mathrm{m} / \mathrm{z}$, $\%): 465.1,100 ; 463.2,84,466.6,21$.
$\mathrm{N}-\{2$ - [2-(2-Iodophenyl) -acetylamino] - ethyl $\}-2-(3,4-$ dichlorophenyl)-acetamide (19): The acid chloride generated from 2-iodophenylacetic acid ( $1.65 \mathrm{~g}, 0.0063$ moles) was added to a solution of 12 (2.03 g, 0.0062 moles) and $\mathrm{Et}_{3} \mathrm{~N}(0.87 \mathrm{~mL}, 0.0063$ moles $)$ to generate the diamide $(0.6311 \mathrm{~g}, 21 \%) .{ }^{3} \mathrm{H}$ NMR $\left(300 \mathrm{MHz}, \mathrm{CDCl}_{3}\right)$ : $\delta$ 3.39 (d, $\left.6 \mathrm{H}, \mathrm{CH}_{2}(\mathrm{C}=\mathrm{O}) \mathrm{NHCH}_{2}\right)$ ), $3.82\left(\mathrm{~s}, 2 \mathrm{H}, \mathrm{Ar} \mathrm{CH}_{2}\right), 5.87$ (br s, $1 \mathrm{H}$, $\mathrm{NH}), 6.42($ br s, $1 \mathrm{H}, \mathrm{NH}), 7.02(\mathrm{t}, 1 \mathrm{H}, \operatorname{ArH}), 7.10$ (d, 1H, ArH) 7.287.41 (m, 4H, ArH), 7.87 (d, 1H, ArH). $\left.{ }^{13} \mathrm{C} \mathrm{NMR} \mathrm{(75} \mathrm{MHz,} \mathrm{CDCl}_{3}\right): \delta$ 29.61, 39.58, 40.64, 42.42, 48.29, 128.71, 128.95, 129.34, 130.59, 130.99, $131.20,131.37,132.63,134.82,137.71,139.83,170.47,171.01$. HPLCMS, retention time $=7.7 \mathrm{~min}$. Theory $(\mathrm{M}+\mathrm{H} \mathrm{m} / \mathrm{z}, \%): 490.9,100 ; 492.9$, 64. Found (M+H m/z, \%): 490.9, 100; 493.0; 60.

N-\{2-[2-(4-Iodophenyl)-acetyla mino]-ethyl $\}-2-(3,4-$ dichlorophenyl)-acetamide (20): The acid chloride generated from 4-iodophenylacetic acid ( $1.33 \mathrm{~g}, 0.0051$ moles) was added to a solution of 12 (1.00 g, 0.0031 moles) and $\mathrm{Et}_{3} \mathrm{~N}(0.90 \mathrm{~mL}, 0.0065$ moles $)$ to generate the diamide $(0.50 \mathrm{~g}, 33 \%) .{ }^{1} \mathrm{H}$ NMR $(300 \mathrm{MHz}, \mathrm{CDCl} 3): \delta 3.33$ $\left.\left(\mathrm{s}, 4 \mathrm{H},(\mathrm{C}=\mathrm{O}) \mathrm{NHCH}_{2}\right)\right), 3.42\left(\mathrm{~s}, 4 \mathrm{H}, \mathrm{Ar} \mathrm{CH}_{2}\right), 6.10$ (br s, $\left.1 \mathrm{H}, \mathrm{NH}\right), 6.34$ (br s, 1H, NH), 6.97 (d, 2H, ArH), 7.08 (d, 1H, ArH), $7.39(\mathrm{~m}, 2 \mathrm{H}$, ArH), 7.67 (d, 2H, ArH). ${ }^{13} \mathrm{C}$ NMR $\left(75 \mathrm{MHz}, \mathrm{CDCl}_{3}\right): \delta 39.97,40.52$, $42.45,42.99,128.75,130.72,131.21,131.21,132.76,134.16,138.0$, $170.88,171.82$. HPLC-MS, retention time $=11.1 \mathrm{~min}$. Theory $(\mathrm{M}+\mathrm{H}$ $\mathrm{m} / \mathrm{z}, \%): 490.9,100 ; 492.9,64$. Found (M+H m/z, \%): 490.9, 100; 492.9; 65 .

General method for reduction of amides $13-20$ : $\mathrm{AlH}_{3}-\mathrm{Et}_{3} \mathrm{~N}$ was prepared as previously described [24]. Briefly, to a $100 \mathrm{~mL}$, 2-necked flask under nitrogen was added $1 \mathrm{M} \mathrm{LiAlH}_{4}(35 \mathrm{~mL}, 0.035 \mathrm{~mol})$ in THF. The solution was stirred, cooled in an ice bath and then treated dropwise with concentrated $\mathrm{H}_{2} \mathrm{SO}_{4}(1.22 \mathrm{~mL}, 0.0439 \mathrm{~mol})$. Neat $\mathrm{Et}_{3} \mathrm{~N}(5.4$ $\mathrm{mL}, 0.039 \mathrm{~mol}$ ) was added to form the reducing agent in situ, and the appropriate amide $(\mathbf{1 3} \mathbf{- 2 0})$ dissolved in freshly distilled THF $(20 \mathrm{~mL})$

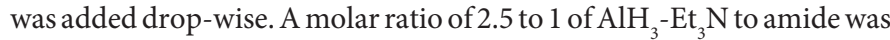
used, and reactions were kept under nitrogen at ambient temperature for $72 \mathrm{~h}$. Mixtures were then poured into ice-cold $2 \mathrm{M} \mathrm{HCl}(15 \mathrm{~mL})$, and concentrated under reduced pressure to $15 \mathrm{~mL} . \mathrm{CH}_{2} \mathrm{Cl}_{2}(30 \mathrm{~mL})$ was added, and the $\mathrm{pH}$ adjusted to $>11$ with aqueous $\mathrm{NaOH}(15 \%)$. Mixtures were extracted with $\mathrm{CH}_{2} \mathrm{Cl}_{2}(3 \times 100 \mathrm{~mL})$, dried $\left(\mathrm{Na}_{2} \mathrm{SO}_{4}\right)$ and filtered. Evaporation under reduced pressure provided oils that were dissolved in absolute ethanol, and converted to salts by addition of $49 \%$ $\mathrm{HBr}$. The free bases were obtained for NMR spectroscopy by treatment of the salts with $15 \% \mathrm{NaOH}(1 \mathrm{~mL})$ and extraction with $\mathrm{CH}_{2} \mathrm{Cl}_{2}(3 \times 2$ $\mathrm{mL})$. After drying $\left(\mathrm{Na}_{2} \mathrm{SO}_{4}\right)$, the $\mathrm{CH}_{2} \mathrm{Cl}_{2}$ was removed under reduced pressure, and the oil was dissolved in the appropriate NMR solvent.

N-[2-(3,4-Dichlorophenyl)-ethyl]-N'-[2-(2-fluorophenyl)ethyl]-ethane-1,2-diamine (4): The acid chloride generated from 2-fluorophenylacetic acid ( $0.55 \mathrm{~g}, 0.0035$ moles) was added to a solution of 12 ( $0.60 \mathrm{~g}, 0.0018$ moles) and $\mathrm{Et}_{3} \mathrm{~N}$ (0.54 mL, 0.0039 moles). The reaction was stirred overnight, purified an oil and the amide 13 (0.694 g, 0.0018 moles) was directly reduced with $\mathrm{AlH}_{3}-\mathrm{Et}_{3} \mathrm{~N}(9 \mathrm{~mL}$, 0.009 moles) and isolated as the dihydrobromide salt $(0.02 \mathrm{~g}, 2 \%) .{ }^{1} \mathrm{H}$ $\operatorname{NMR}\left(300 \mathrm{MHz}, \mathrm{CDCl}_{3}\right): \delta 2.73-3.8\left(\mathrm{~m}, 12 \mathrm{H}, \mathrm{CH}_{2}\right) ; 7.05(\mathrm{~m}, 2 \mathrm{H}, \mathrm{ArH})$; 7.21 (p, 3H, ArH); 7.30 (d, 1H, ArH); 7.35 (d, 1H, ArH). ${ }^{13} \mathrm{C}$ NMR $(75$ $\mathrm{MHz}_{\mathrm{CDCl}}$ ): $\delta 29.81,35.63,48.92,49.14,49.66,50.59,115.12,115.41$, $123.95,127.78,127.88,128.15,130.04,130.28,130.60,130.92,130.99$, 140.51. HPLC-MS, retention time $=6.1 \mathrm{~min}$. Theory $(\mathrm{M}+\mathrm{H} \mathrm{m} / \mathrm{z}, \%)$ : $355.11,100 ; 356.12,19 ; 357.11,64$. Found $(\mathrm{M}+\mathrm{H} \mathrm{m} / \mathrm{z}, \%): 354.98,100$; 356.01, 19; 356.98, 68. Elemental analysis for $\mathrm{C}_{18} \mathrm{H}_{21} \mathrm{Cl}_{2} \mathrm{FN}_{2} \cdot 2 \mathrm{HBr}$ : Theory: C, 41.81; H, 4.48; N, 5.42; Found: C, 41.69; H, 4.43; N, 5.27.

N-[2-(3,4-Dichlorophenyl)-ethyl]- $N^{\prime}$-[2-(3-fluorophenyl)- 
ethyl]-ethane-1,2-diamine (5): Compound 14 (1.30 g, 0.0034 moles) was reduced with $\mathrm{AlH}_{3}-\mathrm{Et}_{3} \mathrm{~N}$ (17 mL, 0.017 moles) and isolated as the dihydrobromide salt $(0.13 \mathrm{~g}, 7 \%) .{ }^{1} \mathrm{H}$ NMR $\left(300 \mathrm{MHz}, \mathrm{D}_{6}\right.$-DMSO): $\delta 2.07(\mathrm{~s}, 2 \mathrm{H}, \mathrm{NH}) ; 2.97\left(\mathrm{t}, 4 \mathrm{H} \mathrm{Ar} \mathrm{CH}_{2}\right) ; 3.28\left(\mathrm{CH}_{2} \mathrm{NHCH}_{2}\right) ; 7.15(\mathrm{t}$, $3 \mathrm{H}, \mathrm{ArH}) ; 7.32$ (d, 1H, ArH); 7.39 (q, 1H, ArH); 7.61 (d, 2H, ArH). ${ }^{13} \mathrm{C}$ NMR (75 MHz, DMSO): $\delta 30.51,60.67,31.16,42.52,47.25,47.42$, $113.64,113.91,115.39,115.67,124.94,129.37,130.55,130.72,130.87$, $131.11,137.96,139.50$. HPLC-MS, retention time $=21.8 \mathrm{~min}$. Theory $(\mathrm{M}+\mathrm{H} \mathrm{m} / \mathrm{z}, \%): 355.1,100 ; 357.1,64 ; 359.1,10$. Found $(\mathrm{M}+\mathrm{H} \mathrm{m} / \mathrm{z}, \%)$ : 354.9, 100; 356.9, 73; 359.0, 10. Elemental analysis for $\mathrm{C}_{18} \mathrm{H}_{21} \mathrm{Cl}_{2} \mathrm{FN}_{2}$ • $2 \mathrm{HBr}$ : Theory: C, 41.81; H, 4.48; N, 5.42; Found: C, 42.07; H, 4.28; N, 5.36 .

N-[2-(3,4-Dichlorophenyl)-ethyl]-N'-[2-(4-fluorophenyl)ethyl]-ethane-1,2-diamine (6): Compound 15 (0.57 g, 0.0015 moles) was reduced with $\mathrm{AlH}_{3}-\mathrm{Et}_{3} \mathrm{~N}$ (7.4 mL, 0.0074 moles) and isolated as the hydrobromide salt $(0.065 \mathrm{~g}, 9 \%) .{ }^{13} \mathrm{C}$ NMR (75 MHz, DMSO): $\delta$ $30.55,30.76,42.57,47.31,47.88,115.23,115.51,129.37,129.55,130.58$, $130.69,130.87,131.08,138.08$. HPLC-MS, retention time $=6.5 \mathrm{~min}$. Theory $(\mathrm{M}+\mathrm{H} \mathrm{m} / \mathrm{z}, \%): 355.1,100 ; 357.1,64 ; 359.1,10$. Found $(\mathrm{M}+\mathrm{H}$ $\mathrm{m} / \mathrm{z}, \%): 355.0,100 ; 357.0,73 ; 359.0,11$.

$\mathrm{N}$-[2-(2-Bromophenyl)-ethyl]-N'-[2-(3,4-dichlorophenyl)ethyl]-ethane-1,2-diamine (7): Compound 16 (0.44 g, 0.0010 moles) was reduced with $\mathrm{AlH}_{3}-\mathrm{Et}_{3} \mathrm{~N}$ (10 mL, 0.010 moles) and isolated as the dihydrobromide salt $(0.32 \mathrm{~g}, 56 \%) .{ }^{1} \mathrm{H}$ NMR $\left(250 \mathrm{MHz}, \mathrm{D}_{2} \mathrm{O}\right): \delta$ 2.96 (t, $2 \mathrm{H}, \mathrm{Ar} \mathrm{CH}_{2}$ ); 3.12 (t, $2 \mathrm{H}, \mathrm{Ar} \mathrm{CH}_{2}$ ); 3.31-3.25 (s and $\mathrm{m}, 8 \mathrm{H}$, $\mathrm{CH}_{2} \mathrm{NHCH}_{2}$ ); 7.25-7.18 (m, 2H, ArH); 7.35-7.34 ( $\mathrm{m}$ and s, $2 \mathrm{H}, \mathrm{ArH}$ ); 7.51-7.46 (s and d, $2 \mathrm{H}, \mathrm{ArH}) ; 7.64(\mathrm{~d}, 1 \mathrm{H}, \mathrm{ArH}) .{ }^{13} \mathrm{C}$ NMR $(62 \mathrm{MHz}$, $\left.\mathrm{CDCl}_{3}\right): \delta 35.61,36.73,48.99,49.14,49.36,50.66,124.57,127.42$, $127.88,128.16,130.06,130.29,130.61,130.74,132.27,132.87,139.36$, 140.44. HPLC-MS, retention time $=20.9 \mathrm{~min}$. Theory $(\mathrm{M}+\mathrm{H} \mathrm{m} / \mathrm{z}, \%)$ : 417.0, 100; 415, 62; 419.0 45. Found ( $\mathrm{M}+\mathrm{H} \mathrm{m} / \mathrm{z}, \%)$ : 416.9, 100; 414.9, 62; 418.9, 44. Elemental analysis for $\mathrm{C}_{18} \mathrm{H}_{21} \mathrm{BrCl}_{2} \mathrm{~N}_{2} \cdot 2 \mathrm{HBr} \bullet 1.5 \mathrm{H}_{2} \mathrm{O}$ : Theory: C, 35.73; H, 4.33; N, 4.63; Found: C, 35.72; H, 4.10; N, 4.69.

$\mathrm{N}$-[2-(3-Bromophenyl)-ethyl]-N'-[2-(3,4-dichlorophenyl)ethyl]-ethane-1,2-diamine (8): Compound 17 (0.66 g, 0.0015 moles) was reduced with $\mathrm{AlH}_{3}-\mathrm{Et}_{3} \mathrm{~N}(7.5 \mathrm{~mL}, 0.0075$ moles $)$ and isolated as the dihydrobromide salt $(0.38 \mathrm{~g}, 44 \%) .{ }^{1} \mathrm{H}$ NMR $\left(300 \mathrm{MHz}, \mathrm{D}_{2} \mathrm{O}\right): \delta$ 3.02 (s, $\left.4 \mathrm{H}, \mathrm{Ar} \mathrm{CH}_{2}\right) ; 3.42$ (s, 8 $\mathrm{H} \mathrm{CH}_{2} \mathrm{NHCH}_{2}$ ); 7.52-7.30 (m, 7H, ArH). ${ }^{13} \mathrm{C}$ NMR $\left(75 \mathrm{MHz}, \mathrm{CDCl}_{3}\right): \delta 35.41,36.51,48.77,48.90,49.17,124.39$, $127.29,127.73,128.53,129.85,130.14,130.47,130.60,130.73,132.06$, $132.69,139.21,140.4$. HPLC-MS, retention time $=22.1 \mathrm{~min}$. Theory $(\mathrm{M}+\mathrm{H} \mathrm{m} / \mathrm{z}, \%): 417.0,100 ; 415.0,62 ; 419.0,45$. Found $(\mathrm{M}+\mathrm{H} \mathrm{m} / \mathrm{z}, \%)$ : $416.9,100 ; 414.9,60 ; 418.9$, 45. Elemental analysis for $\mathrm{C}_{18} \mathrm{H}_{21} \mathrm{BrCl}_{2} \mathrm{~N}_{2}$ • 2 HBr: Theory: C, 37.40; H, 4.01; N, 4.85; Found: C, 37.62; H, 3.96; N, 4.78 .

$N$-[2-(4-Bromophenyl)-ethyl]-N'-[2-(3,4-dichlorophenyl)ethyl]-ethane-1,2-diamine (9): Compound 18 (0.4 g, 0.0009 moles) was reduced with $\mathrm{AlH}_{3}-\mathrm{Et}_{3} \mathrm{~N}$ (4.52 mL, 0.0045 moles) and isolated as the salt $(0.13 \mathrm{~g}, 25 \%) .{ }^{1} \mathrm{H}$ NMR $\left(500 \mathrm{MHz}, \mathrm{CDCl}_{3}\right) \delta 2.72-2.76$ (s and $\mathrm{m}$, $\left.8 \mathrm{H}, \mathrm{CH}_{2} \mathrm{NHCH}_{2}\right) ; 2.85$ (t, $4 \mathrm{H} \mathrm{Ar} \mathrm{CH}$ ); 7.04-7.06 (d of d, $\left.1 \mathrm{H}, \mathrm{ArH}\right) ; 7.09$ (d, 2H, ArH); 7.32 (d, 1H, ArH); 7.37 (d, 1H, ArH); 7.42 (d, 2H, ArH). ${ }^{13} \mathrm{CNMR}\left(75 \mathrm{MHz}, \mathrm{CDCl}_{3}\right.$ ): $\delta 29.69,32.42,35.63,35.9,49.18,50.89$, $51.13,119.88,128.17,128.43,128.69,129.73,130.05,130.44,130.60$, $131.47,132.26,139.10$. HPLC-MS, retention time $=22.4 \mathrm{~min}$. Theory $(\mathrm{M}+\mathrm{H} \mathrm{m} / \mathrm{z}, \%): 417.0,100 ; 415.0,62 ; 419.0,45$. Found $(\mathrm{M}+\mathrm{H} \mathrm{m} / \mathrm{z}, \%)$ : 416.9, 100; 414.9, 60; 418.9, 43. Elemental analysis for $\mathrm{C}_{18} \mathrm{H}_{21} \mathrm{BrCl}_{2} \mathrm{~N}_{2}$ - $1.75 \mathrm{HBr} \bullet 0.25 \mathrm{H}_{2} \mathrm{O}$ : Theory: C, 38.45; H, 4.17; N, 4.98; Found: C, 38.44; H, 4.09; N, 4.65.

N-[2-(3,4-Dichlorophenyl)-ethyl]-N'-[2-(2-iodophenyl)-ethyl]- ethane-1,2-diamine (10): Compound 19 (0.31 g, 0.0006 moles) was reduced with $\mathrm{AlH}_{3}-\mathrm{Et}_{3} \mathrm{~N}(3 \mathrm{~mL}, 0.003$ moles $)$ at $0{ }^{\circ} \mathrm{C}$ and isolated as the salt (0.12 g, 32\%). ${ }^{1} \mathrm{H}$ NMR (300 MHz, $\mathrm{D}_{6}$-DMSO): $\delta 2.9-3.3(\mathrm{~m}, 8 \mathrm{H}$ $\left.\mathrm{CH}_{2}\right) 7.30$ (s, 1H, ArH), 7.38 (d, 2H, ArH) 7.63 (s, 1H, ArH) 7.89 (d, $1 \mathrm{H}, \mathrm{ArH}) 8.98(\mathrm{~d}, 3 \mathrm{H}, \mathrm{ArH}) .{ }^{13} \mathrm{C} \mathrm{NMR}\left(75 \mathrm{MHz}, \mathrm{CDCl}_{3}\right): \delta 30.47,36.36$, $42.53,46.59,47.27,101.74,128.69,128.83,129.21,129.39,129.59$, $129.90,130.71,130.89,131.11,137.99,139.37$. HPLC-MS, retention time $=24.9 \mathrm{~min}$. Theory $(\mathrm{M}+\mathrm{H} \mathrm{m} / \mathrm{z}, \%): 463.0,100 ; 464.0,20 ; 465.0$, 64. Found $(\mathrm{M}+\mathrm{H} \mathrm{m} / \mathrm{z}, \%): 462.9,100 ; 464.0,21 ; 464.9$, 63. Elemental analysis for $\mathrm{C}_{18} \mathrm{H}_{21} \mathrm{ICl}_{2} \mathrm{~N}_{2} \bullet 1.75 \mathrm{HBr} \bullet 0.75 \mathrm{H}_{2} \mathrm{O}$ : Theory: $\mathrm{C}, 35.48 ; \mathrm{H}$, 4.22; N, 4.47; Found: C, 35.48; H, 4.16; N, 4.35 .

$N$-[2-(3,4-Dichlorophenyl)-ethyl]-N'-[2-(4-iodophenyl)-ethyl]ethane-1,2-diamine(11): Compound 20 (0.25 g, 0.0005 moles), was reduced with $\mathrm{AlH}_{3}-\mathrm{Et}_{3} \mathrm{~N}(3 \mathrm{~mL}, 0.003$ moles $)$ at $0{ }^{\circ} \mathrm{C}$ and isolated as the dihydrobromide salt $(0.052 \mathrm{~g}, 17 \%) .{ }^{1} \mathrm{H}$ NMR $\left(500 \mathrm{MHz}, \mathrm{CDCl}_{3}\right)$ : $\delta$ 2.75-2.88 (m, 12 $\left.\mathrm{H}, \mathrm{CH}_{2}\right), 7.00(\mathrm{dd}, 1 \mathrm{H}, \mathrm{ArH}), 7.2(\mathrm{~d}, 2 \mathrm{H}, \mathrm{ArH}), 7.33$ (t, 2H, ArH), 7.36-7.38 (m, 1H, ArH), $7.63(\mathrm{~d}, 1 \mathrm{H}, \mathrm{ArH}) .{ }^{13} \mathrm{C} \mathrm{NMR}$ $\left(75 \mathrm{MHz} \mathrm{CDCl}_{3}\right): \delta 35.45,35.78,50.47,50.67,53.32,58.31,128.09$, $128.69,130.00,130.22,130.50,130.71,132.19,137.38,139.57,140.27$. HPLC-MS, retention time $=24.9 \mathrm{~min}$. Theory $(\mathrm{M}+\mathrm{H} \mathrm{m} / \mathrm{z}, \%): 463.0$, $100 ; 464.0,20 ; 465.0,64$. Found $(\mathrm{M}+\mathrm{H} \mathrm{m} / \mathrm{z}, \%): 462.9,100 ; 464.0,21$ 464.9, 67. Elemental analysis for $\mathrm{C}_{18} \mathrm{H}_{21} \mathrm{ICl}_{2} \mathrm{~N}_{2} \bullet 2 \mathrm{HBr}$ : Theory: $\mathrm{C}, 34.59$; $\mathrm{H}, 3.71$; N, 4.48; Found: C, 34.36; H, 3.78; N, 4.44.

\section{Lipophilicity Measurements}

Computational method: Specific algorithms for calculating ClogP utilized fragment-based methods developed by the Medicinal Chemistry Project and BioByte [25] contained as a subroutine in ChemDraw 9.0 (CambridgeSoft Corporation, Cambridge, MA).

Reverse-phase HPLC method: These procedures were performed as described by Minick et al. [26]. The HPLC equipment consisted of Waters M6000A pumps and a Waters 490E programmable multiwavelength detector. The guard column (C18) and main column (Econosil C8, $4.6 \mathrm{~mm} \mathrm{X} 10 \mathrm{~cm}$ ) were from Alltech Applied Science (State College, PA). The organic phase was methanol containing $0.25 \%(\mathrm{v} / \mathrm{v}) n$-octanol and the aqueous phase was $0.02 \mathrm{M}$ MOPS buffer containing $0.12 \%(\mathrm{v} / \mathrm{v}) \mathrm{n}$-decylamine $(\mathrm{pH}$ 7.5). Multiple different organic / aqueous compositions were utilized at a flow rate of $2 \mathrm{~mL} /$ min. Ligand samples were dissolved in $1 \mathrm{~mL}$ of the organic phase, and 3 injections each of samples and standards at 3 different concentrations of organic phase were performed. The void volume was determined with urea, detected at UV $=214 \mathrm{~nm}$. All other compounds were detected at 280 and $254 \mathrm{~nm}$. The $\kappa \times$ value for all injected samples and standards was calculated with the following formula: $\kappa \times \times_{v}=$ (retention time of compound / dead time) - 1. Then a graph of $\lambda$ o $\gamma \kappa \times$ versus fraction methanol was generated. The data for standards and ligand samples were fit to a linear equation, and the intercept was determined which is the $\lambda$ o $\gamma \kappa \times \sqrt{ }$. Next, a curve was generated for the standards by plotting the known $\log$ PC values versus the experimental $\lambda o \gamma \kappa \times_{\downarrow}$ and a linear equation $\left(\mathrm{y}=0.92 \mathrm{x}+0.90 ; \mathrm{r}^{2}=0.94\right)$ was generated where $\mathrm{y}=$ $k^{\prime}{ }_{w}$ calculated and $\mathrm{x}=\log k^{\prime}$. Then, $k^{\prime}{ }_{w}$ was determined for the samples from the equation for the standard curve and the intercept $\log k^{\prime}{ }_{\gamma}$ of the samples.

\section{$\sigma_{1}$ Receptor Binding Assays}

Binding assays were performed using $1.0 \mathrm{nM}\left[{ }^{3} \mathrm{H}\right](+)$-pentazocine and membranes prepared from fresh-frozen guinea pig brains as previously described $[27,28]$ with minor modifications. Non-specific binding was defined by haloperidol $(10.0 \mu \mathrm{M})$, each assay tube contained $0.24 \mathrm{mg}$ protein, and assays were performed in Tris- $\mathrm{HCl}$ buffer $(50 \mathrm{mM}$, 
$\mathrm{pH} 7.4$ ) at $37^{\circ} \mathrm{C}$ for $150 \mathrm{~min}$. Assays were terminated by the addition of ice-cold buffer, and rapid filtration through Whatman GF/B glass fiber filters that had been presoaked in $0.5 \%$ polyethylenimine. Filter papers were then washed with ice-cold buffer $(3 \times 5 \mathrm{~mL})$, soaked in cocktail, dark-adapted overnight and then counted for tritium. Test compounds were dissolved in the minimum amount of ethanol, and assay buffer was added to make a concentrated stock $\left(1 \times 10^{-3} \mathrm{M}\right)$ that was used to prepare serial dilutions in buffer. Ligand concentrations in the assays ranged from 1000 to $0.1 \mathrm{nM}$. The final concentration of ethanol in any assay tube never exceeded $0.5 \%$, an amount that did not affect $\left[{ }^{3} \mathrm{H}\right](+)$-pentazocine binding in control studies. The $\mathrm{IC}_{50}$ and $K_{\mathrm{i}}$ values were determined in two to four assays, each performed in duplicate, by non-linear regression of binding data using curve-fitting programs Prism 4.0b (Graph-Pad Software, San Diego, CA) and Radlig 6.0 (Biosoft, Inc., Ferguson, $\mathrm{MO}$ ). $K_{\mathrm{i}}$ values were derived from $\mathrm{IC}_{50}$ data by the Cheng-Prusoff relationship [29] using an input $K_{d}$ of $2.3 \mathrm{nM}$ for $\left[{ }^{3} \mathrm{H}\right](+)$-pentazocine [28].

\section{Results and Discussion}

The construction of the eight halogenated $N, N^{\prime}$ diphenethylethylenediamines (4 - 11) involved linear synthesis from amide $\mathbf{1 2}$ as a common precursor (Figure 2). The synthesis of $\mathbf{1 2}$ was accomplished by a two-step, activation - amidation process. Several methods for activation of 3,4-dichlorophenylacetic acid were explored, including conversion of the acid to either an active ester or to an anhydride with DCC, followed by amide formation by coupling with anhydrous ethylenediamine. Based upon the initial yields and ease of performance, activation using $\mathrm{N}$-hydroxyphthalimide and DCC proved to be the method of choice. This reaction was conducted six times on a 5 - 6 gram scale of the carboxylic acid, and yielded $\mathbf{1 2}$ as the white hydrobromide salt in a reproducible $31 \pm 6 \%$ yield.

The formation of diamides $\mathbf{1 3}$ - $\mathbf{2 0}$ also was investigated using two different routes. The first involved DCC-mediated coupling of 12 with the carboxylic acid, and the second involved coupling of 12 with the carboxylic acid chloride. Synthesis via the carboxylic acid chloride route was advantageous because the reaction side products are soluble in water while the diamides readily precipitate. Thus, the final diamide products were obtained in high purity by simple filtration, and procedural issues involving removal of DCU during the alternative route were avoided. Isolated yields for diamides 13 - 20 ranged between $21-93 \%$.

The final target compounds $\mathbf{4}$ - $\mathbf{1 1}$ were prepared by reduction of the corresponding diamides. Exploratory attempts to accomplish the transformations using $\mathrm{LiAlH}_{4}$ at $0{ }^{\circ} \mathrm{C}$ resulted in complex product

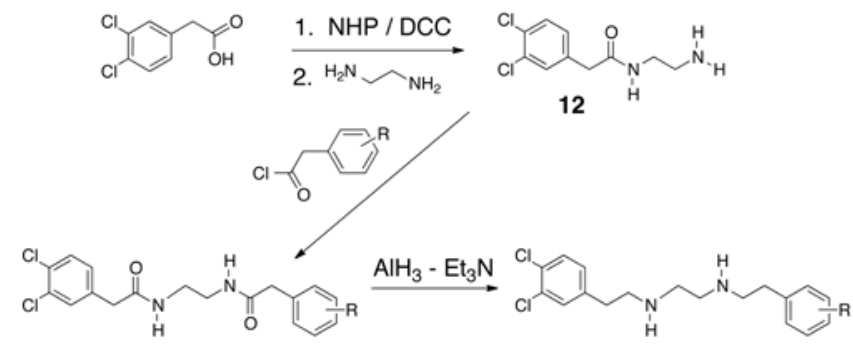

13, $\mathrm{R}=2-\mathrm{F} \quad 16, \mathrm{R}=2-\mathrm{Br}$ $14, R=3-\mathrm{F}$ 17, $R=3-\mathrm{Br} 19, \mathrm{R}=2-1$
$15, \mathrm{R}=4-\mathrm{F}$ 18, $\mathrm{R}=4-\mathrm{Br}, 20, \mathrm{R}=4-1$

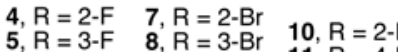
$\begin{array}{lll}5, R=3-F & 8, R=3-B r & 11, R=4-I\end{array}$

Figure 2: Synthetic route to halogenated $N, N^{\prime}$-diphenethylethylenediamines $4-11$. mixtures as a consequence of loss of aromatic halogens. Subsequently, the more selective aluminum hydride-triethylamine $\left(\mathrm{AlH}_{3}-\mathrm{Et}_{3} \mathrm{~N}\right)$ reducing agent developed by Cha and Brown [24] was investigated. The $\mathrm{AlH}_{3}-\mathrm{Et}_{3} \mathrm{~N}$ was prepared in situ, and a molar ratio of 2.5 to 1 of $\mathrm{AlH}_{3}-\mathrm{Et}_{3} \mathrm{~N}$ to amide was employed. Reactions were allowed to proceed at room temperature under nitrogen for 2.5 days. Isolated yields of the fluoro analogs 4 - $\mathbf{6}$ as the dihydrobromide salts were low, $<10 \%$, but the procedure was straightforward. Improved isolated yields, 25 $56 \%$, were obtained for the bromo congeners $7-9$. For the iodinated diamides 19 and $20, \mathrm{AlH}_{3}-\mathrm{Et}_{3} \mathrm{~N}$ reductions at room temperature gave mixtures of the iodinated and the deiodinated derivatives. However, deiodination was not observed when the reactions were kept at $0{ }^{\circ} \mathrm{C}$. Thus, the 2- and 4-iodophenethyl derivatives, 10 and 11, were at hand in yields of $32 \%$ and $17 \%$, respectively. Attempts to prepare $\mathbf{1 0}$ and $\mathbf{1 1}$ from bromo analogs 7 and 9 by copper-assisted iodine for bromine exchange, or through stannylated intermediates, were unsuccessful.

The $\log k_{w}^{\prime}$ values for representative isomeric $N, N^{\prime}$ diphenethylethylenediamines were determined experimentally by a reverse-phase HPLC method [26], as well as calculated using ClogP [25] (Table 1). When comparing the experimental $k_{w}^{\prime}$ values for compounds with similar structures, the expected order of lipophilicity is fluoro- $<$ bromo- $<$ iodo- based on known lipophilicity constants. This relationship held true for analogs 6, 9 and $\mathbf{1 1}$ having the three different halogens in the 4-position (Table 1). There is no significant difference in $k^{\prime}$ between the 2-bromophenyl and 3-bromophenyl derivatives 7 and 8; however, the value for the 4-bromophenyl derivative 9 was $0.22-0.25$ units higher. Considering that this is an HPLC method, it appears that the interaction of these isomers with the stationary phase promotes subtle differences. The experimentally determined $k^{\prime}$ values ranged from $2.54-3.71$ while the calculated Clog P values ranged from $5.03-6.01$. This difference in magnitude can be explained by the fact that the $\mathrm{pH}$ of the aqueous component of the HPLC solvent system is 7.5 , and it is known that the computer program does not adjust for the protonation status of the amines.

To determine the ability of compounds $\mathbf{4}-\mathbf{1 1}$ to bind to $\sigma_{1}$ receptors, competition assays against $\left[{ }^{3} \mathrm{H}\right](+)$-pentazocine were performed using established methods in guinea pig brain membranes [27,28]. Binding parameters are summarized in (Table 1). Within each individual halogen series, the 3- and 4- substituted derivatives displayed higher binding affinities than the 2-substituted derivative. This difference is pronounced in relation to the size of the halogen. The 2-fluoro substituted ligand 4 shows only a 1.5 -fold lower $\sigma_{1}$ receptor affinity than positional isomers $\mathbf{5}$ and $\mathbf{6}$. By contrast, the 2-bromo substituted ligand 7 exhibits 2.7 fold lower affinity than isomers $\mathbf{8}$ and $\mathbf{9}$. Similarly, 2-iodo analog 10 suffers a 2.7 -fold loss of affinity compared to the 4 -iodo isomer 11. These data indicate that $\sigma_{1}$ receptor binding in this series is sensitive to steric bulk at the 2-position. The apparent affinity $\left(K_{\mathrm{i}}\right)$ values for the 3-and 4-substituted bromo derivatives $\mathbf{8}$ and 9 are quite similar to those observed for the 3 - and 4-substituted fluoro derivatives 5 and 6. Conversely, the 4 -substituted iodo derivative $\mathbf{1 1}$ has a 2 -fold poorer $K_{\mathrm{i}}$ value than either one of compounds 6 or 9 . In keeping with this trend, 10 displays lower affinity than either $\mathbf{4}$ or $\mathbf{7}$. Thus, fluoro and bromo substituents impart enhanced $\sigma_{1}$ receptor binding affinity as compared to the corresponding iodinated derivatives for this series of ligands.

\section{Conclusions}

The synthesis and evaluation of $\sigma_{1}$ receptor binding diphenethylethylenediamines with a 3,4 dichlorophenyl moiety and a halogenated phenyl ring was explored. The structural modifications 
Citation: Fitzsimmons JM, Lever JR, Lever SZ (2011) Synthesis and $\sigma_{1}$ Receptor Binding of Halogenated N,N'-Diphenethylethylenediamines. Medchem 1:102. doi:10.4172/2161-0444.1000102

Page 6 of 7

\begin{tabular}{|c|c|c|c|c|c|}
\hline \multirow[b]{2}{*}{ Compound } & \multicolumn{2}{|c|}{ Lipophilicity } & \multicolumn{3}{|c|}{$\sigma_{1}$ Receptor Binding Parameters } \\
\hline & $k_{w}^{\prime}$ & ClogP & $\mathrm{IC}_{50}(\mathrm{nM})$ & $K_{\mathrm{i}}(\mathrm{nM})$ & Hill Slope \\
\hline $4,2-F ; n=4$ & ND & 5.03 & $17.48 \pm 0.31$ & $12.08 \pm 0.22$ & $-1.07 \pm 0.01$ \\
\hline $5,3-F ; n=4$ & ND & 5.03 & $11.21 \pm 1.29$ & $74 \pm 0.89$ & $-1.13 \pm 0$ \\
\hline $6,4-F ; n=4$ & $2.54 \pm 0.05$ & 5.03 & $11.34 \pm 2.02$ & $7.83 \pm 1.39$ & $-1.21 \pm 0.05$ \\
\hline $7,2-B r ; n=3$ & $3.09 \pm 0.05$ & 5.75 & $26.62 \pm 3.95$ & $18.39 \pm 2.73$ & $-0.78 \pm 0.03$ \\
\hline $8,3-B r ; n=3$ & $3.06 \pm 0.07$ & 5.75 & $9.14 \pm 0.85$ & $6.35 \pm 0.59$ & $-1.02 \pm 0.15$ \\
\hline $9,4-B r ; n=3$ & $3.31 \pm 0.08$ & 5.75 & $9.85 \pm 3.92$ & $6.80 \pm 2.71$ & $-1.00 \pm 0.02$ \\
\hline 10, 2-I; n = 2 & ND & 6.01 & $62.47 \pm 0.96$ & $43.15 \pm 0.66$ & $-1.12 \pm 0.03$ \\
\hline $11,4-1 ; n=4$ & $3.71 \pm 0.05$ & 6.01 & $22.89 \pm 0.94$ & $5.82 \pm 0.65$ & $-1.59 \pm 0.08^{*}$ \\
\hline
\end{tabular}

ND $=$ not determined. Binding parameters are means \pm SEM for two to four assays, each performed in duplicate, $\mathrm{n}=$ number of trials. * Significantly different from unity

Table 1: Lipophilicity measurements and $\sigma_{1}$ receptor binding parameters.

provided differences in lipophilicity, and influenced $\sigma_{1}$ receptor binding. Affinity was moderately sensitive to steric bulk at the 2- position. Bromine or fluorine substituents at a given position gave higher $\sigma_{1}$ receptor binding affinity than an iodine substituent. The 8 -atom spacer between the hydrophobic (aromatic) groups in compounds $\mathbf{4 - 1 1}$ is consistent models described by Glennon, Ablordeppey and colleagues $[18,30]$, which indicate that $7-10$ atoms are needed between the primary and secondary hydrophobic regions of the postulated pharmacophore to confer high $\sigma_{1}$ receptor binding affinity. Lipophilicity measurements, coupled with $\sigma_{1}$ receptor binding affinities, suggest that ligands from this active series, such as 3 -fluoro analog 5 , may be good candidates for in vivo studies.

\section{Acknowledgments}

We thank the National Institutes of Health, National Cancer Institute $(\mathrm{NIH} \mathrm{NCl}$ P50 CA 103130: Center for Single Photon-Emitting Cancer Imaging Agents) and National Institute on Drug Abuse (1RC1 DA028477: Development of Anti-Cocaine Medications) for partial support of this research, and for graduate (JMF) research fellowships. We also acknowledge resources provided by Harry S. Truman Memorial Veterans' Hospital, the University of Missouri Life Sciences Mission Enhancement Program, and NSF CHE-95-31247 and NIH 1S10RR11962-01 grant awards for NMR instrumentation.

\section{References}

1. Matsumoto RR, Liu Y, Lerner M, Howard EW, Brackett DJ (2003) $\sigma$ receptors: Potential medications development target for anti-cocaine agents. Eur $\mathrm{J}$ Pharmacol 469: 1-12.

2. Aydar E, Palmer CP, Djamgoz MB (2004) Sigma receptors and cancer: Possible involvement of ion channels. Cancer Res 64: 5029-5035.

3. Monnet FP, Maurice T (2006) The sigma1 protein as a target for the nongenomic effects of neuro(active)steroids: Molecular, physiological, and behavioral aspects. J Pharmacol Sci 100: 93-118.

4. Maurice T, Su TP (2009) The pharmacology of sigma-1 receptors. Pharmacol Ther 124: 195-206

5. Cobos EJ, Entrena JM, Nieto FR, Cendán CM, Del Pozo E (2006) Pharmacology and therapeutic potential of sigma, receptor ligands. Curr Neuropharmacol 6 : 344-366

6. Hayashi T, Su TP (2008) An update on the development of drugs for neuropsychiatric disorders: Focusing on the $\sigma_{1}$ receptor ligand. Expert Opin Ther Targets 12: 45-58.

7. Hayashi T, Tsai SY, Mori T, Fujimoto M, Su TP (2011) Targeting ligand-

operated chaperone sigma-1 receptors in the treatment of neuropsychiatric disorders. Expert Opin Ther Targets 15: 557-577.

8. Ishikawa M, Hashimoto $K$ (2010) The role of sigma-1 receptors in the pathophysiology of neuropsychiatric diseases. J Receptor Ligand Channel Res 3: 25-36.

9. Valade A, Cross SB, Brown C, Detrait E, Ene D, et al. (2011) Discovery of novel selective sigma-1 ligands as cognitive enhancers. Med Chem Commun 2: 655-660.

10. Sabino V, Cottone P, Blasio A, Iyer MR, Steardo L, Rice KC, et al. (2011) Activation of $\sigma$-receptors induces binge-like drinking in Sardinian alcoholpreferring rats. Neuropsychopharmacol 36: 1207-1218.

11. Matsumoto RR (2009) Targeting sigma receptors: novel medication development for drug abuse and addiction. Expert Rev Clin Pharmacol 2: 351 358 .

12. Matsumoto RR, Shaikh J, Wilson LL, Vedam S, Coop A (2008) Attenuation of methamphetamine-induced effects through antagonism of sigma receptors: evidence from in vivo and in vitro studies. Eur J Neuropsychopharmacol 18 : 871-881.

13. Rodvelt KR, Lever SZ, Lever JR, Blount LR, Fan K-H, et al. (2011) SA4503 attenuates cocaine-induced hyperactivity and enhances methamphetamine substitution for a cocaine discriminative stimulus. Pharmacol Biochem Behav 97: 676-682.

14. Rodvelt KR, Oelrichs CE, Blount LR, Fan KH, Lever SZ, et al. (2011) The sigma receptor agonist SA4503 both attenuates and enhances the effects of methamphetamine. Drug Alcohol Depend 116: 203-210.

15. Narayanan S, Mesangeau C, Poupaert JH, McCurdy CR (2011) Sigma receptors and cocaine abuse. Curr Top Med Chem 11: 1128-1150.

16. Matsumoto RR, Pouw B, Mack AL, Daniels A, Coop A (2007) Effects of UMB24 and (+/-)-SM 21, putative sigma2-preferring antagonists, on behavioral toxic and stimulant effects of cocaine in mice. Pharmacol Biochem Behav 86: 86-91.

17. Fan KH, Lever JR, Lever SZ (2011) Effect of structural modification in the amine portion of substituted aminobutyl-benzamides as ligands for binding $\sigma$ and $\sigma_{2}$ receptors. Bioorg Med Chem 19: 1852-1859.

18. Glennon RA (2005) Pharmacophore identification for sigma-1 $\left(\sigma_{1}\right)$ receptor binding: application of the deconstruction - reconstruction - elaboration approach. Mini Rev Med Chem 5: 927-940.

19. Foster A, Wu H, Chen W, Williams W, Bowen WD, et al. (2003) 1,4-Dibenzylpiperazines possess anticocaine activity. Bioorg Med Chem Let 13: $749-751$.

20. Matsumoto RR, Potelleret FH, Mack A, Pouw B, Zhang $Y$, et al. (2004) Structure-activity comparison of $Y Z-069$, a novel sigma ligand, and four analogs in receptor binding and behavioral studies. Pharmacol Biochem Behav 77: $775-781$

21. Nahas RI, Lever JR, Lever SZ (2008) Synthesis and structure-activity relationships of $\mathrm{N}$-(3-phenylpropyl)-N'-benzylpiperazines: potent ligands for $\sigma_{1}$ and $\sigma_{2}$ receptors. Bioorg Med Chem 16: 755-761.

22. Hashimoto K, Ishiwata K (2006) Sigma receptor ligands: possible application as therapeutic drugs and as radiopharmaceuticals. Curr Pharm Des 12: $3857-$ 3876.

23. Collier TL, Waterhouse RN, Kassiou M (2007) Imaging sigma receptors: applications in drug development. Curr Pharm Design 13: 51-72.

24. Cha JS, Brown HC (1993) Reaction of aluminum hydride-triethylamine complex with selected organic compounds containing representative functional groups. J Org Chem 58: 3974-3979.

25. Leo AJ (1993) Calculating log Poct from structures. Chem Rev 93: 1281-1306.

26. Minick DJ, Frenz JH, Patrick MA, Brent DA (1988) A comprehensive method for determining hydrophobicity constants by reversed-phase high-performance liquid chromatography. J Med Chem 31: 1923-1933.

27. DeHaven-Hudkins DL, Fleissner LC, Ford-Rice FY (1992) Characterization of the binding of $\left[{ }^{3} \mathrm{H}\right](+)$-pentazocine to sigma recognition sites in guinea pig brain. Eur J Pharmacol 227: 371-378.

28. Lever JR, Gustafson JL, Xu R, Allmon RL, Lever SZ (2006) $\sigma_{1}$ and $\sigma_{2}$ receptor binding affinity and selectivity of SA4503 and fluoroethyl SA4503. Synapse 59 : 350-358. 
Citation: Fitzsimmons JM, Lever JR, Lever SZ (2011) Synthesis and $\sigma_{1}$ Receptor Binding of Halogenated N,N'-Diphenethylethylenediamines. Medchem 1:102. doi:10.4172/2161-0444.1000102

29. Cheng Y, Prusoff WH (1973) Relationship between the inhibition constant (K) and the concentration of inhibitor which causes 50 per cent inhibition $\left(\mathrm{IC}_{50}\right)$ of an enzymatic reaction. Biochem Pharmacol 22: 3099-3108
30. Ablordeppey SY, Fischer JB, Law H, Glennon RA (2002) Probing the proposed phenyl-A region of the sigma-1 receptor. Bioorg Med Chem 10: 2759-2765. 